\title{
Solving Diffusion Equation Using a New Multiquadric Quasi-interpolation
}

\author{
Junying Cao ${ }^{*}, 1$,Ziqiang Wang ${ }^{2}$ \\ ${ }^{*}$ C College of Science,Guizhou Minzu University,550025,Guiyang,China. \\ Email: caojunying1000@126.com \\ 2 College of Science,Guizhou Minzu University,550025,Guiyang,China. \\ Email:wangzq@1sec.cc.ac.cn
}

\begin{abstract}
In this paper, a new univariate quasi-interpolation operator is presented by means of construction way with cubic Multiquadric functions. It possesses univariate cubic polynomial reproduction property, quasi convexity-preserving and shapepreserving of order 4 properties, and a higher convergence rate. First, the quasi-interpolation operator $L_{R}(x)$ is applied to approximate the derivative of order $m=1,2,3$ and its approximation capacity is obtained, i.e., $C_{0} h(h+c)^{3-m}+C_{1} c^{2}$.

Second, it is used to construct numerical schemes to solve the diffusion equation. Using the derivative of the quasi-interpolation to approximate the spatial derivative of the differential equation. And applying Crank-Nicolson scheme and back Euler scheme to approximate the temporal derivative of the differential equation. And as $c=O\left(h^{2}\right)$, the computational accuracy of the scheme is both $O\left(\Delta t^{2}+h^{2}\right)$ and $O\left(\Delta t+h^{2}\right)$ respectively. Finally, some numerical examples is given to verify the scheme for the onedimensional diffusion equation. The numerical results show that the numerical solution are very close to the exact solution.
\end{abstract}

Keywords-Multiquadric quasi-interpolation;diffusion equation; shape-preserving property; approximation capacity

\section{INTRODUCTION}

With the development of radial basis functions, more and more researchers construct interpolation functions with them, and have obtained better results. For example,[1,2] introduced some interpolation functions by using radial basis functions. In particular, the Multiquadric function of first degree was proposed in [3], which performed well in many fields, and Franke [4] also showed that the interpolation functions in virtue of Multiquadric functions were the best in accuracy and efficiency by performing many numerical experiments. However, when the number of interpolation points is very large, the interpolation matrix might be illconditioning. Compared with interpolation, the quasiinterpolation method not only can avoid the ill-conditioning problem, but also possesses the polynomial reproduction property and better shape-preserving properties. On the basis of these advantages of quasi-interpolation method, researching how to construct a quasi-interpolation operator with better properties to non-uniformly distributed data has been a hot topic recently [5,6,7]. For a good performance of the Multiquadric quasi-interpolation method scheme, many researchers have employed the Multiquadric quasi- interpolation scheme as a numerical method to solve partial differential equations $[8,9,10,11]$.

In this article, we develop a method, namely, applying a kind of univariate Multiquadric quasi-interpolation to solve diffusion equation. The present method can be used to deal with the complicated boundary conditions and the initial conditions with scattered data.

In our methods, we use the derivative of the Multiquadric quasi-interpolation to approximate the spatial derivative of the differential equations and employ Crank-Nicolson and back Euler scheme for the approach of the temporal derivative.

A. Constructing a quasi-interpolation operator by shift of cubic multiquadric function to scattered data

Suppose $f(x)$ is smooth enough, we will construct a quasiinterpolation operator $L_{R}(x)$ by shifts of cubic multiquadric function $\phi_{j}(x)=\left[\left(x-x_{j}\right)^{2}+c^{2}\right]^{\frac{3}{2}}$ to scattered points $\left\{x_{j}\right\}_{j=0}^{n}$ and data points $\left\{\left(x_{j}, f\left(x_{j}\right)\right)\right\}_{0 \leq j \leq n}$, where $c$ is a positive constant and

$$
\begin{aligned}
& A=x_{0}<x_{1}<x_{2}<\cdots<x_{n}=B, \\
& h=\max _{0 \leq j \leq n-1}\left(x_{j+1}-x_{j}\right), \\
& L_{R}(x)=f\left(x_{0}\right) \alpha_{0}(x)+f\left(x_{1}\right) \alpha_{1}(x)+f\left(x_{2}\right) \alpha_{2}(x) \\
& +f\left(x_{3}\right) \alpha_{3}(x)+\sum_{j=4}^{n-4} f\left(x_{j}\right) \alpha_{j}(x) \\
& +f\left(x_{n-3}\right) \alpha_{n-3}(x)+f\left(x_{n-2}\right) \alpha_{n-2}(x) \\
& +f\left(x_{n-1}\right) \alpha_{n-1}(x)+f\left(x_{n}\right) \alpha_{n}(x),
\end{aligned}
$$

where

$$
\begin{aligned}
\alpha_{j}= & \frac{\omega_{j}(x)-\omega_{j+1}(x)}{2\left(x_{j+2}-x_{j}\right)\left(x_{j+1}-x_{j}\right)}-\frac{\omega_{j-1}(x)-\omega_{j}(x)}{2\left(x_{j+1}-x_{j}\right)\left(x_{j+1}-x_{j-1}\right)} \\
- & \frac{\omega_{j-1}(x)-\omega_{j}(x)}{2\left(x_{j+1}-x_{j-1}\right)\left(x_{4}-x_{3}\right)}-\frac{\omega_{j-2}(x)-\omega_{j-1}(x)}{2\left(x_{j}-x_{j-1}\right)\left(x_{j}-x_{j-2}\right)}, \\
\alpha_{0}(x)= & \frac{1}{2}-\frac{x-x_{0}}{2\left(x_{1}-x_{0}\right)}+\frac{\left(x-x_{0}\right)\left(x-x_{1}\right)}{2\left(x_{2}-x_{0}\right)\left(x_{1}-x_{0}\right)} \\
- & \frac{\left(x-x_{0}\right)\left(x-x_{1}\right)\left(x-x_{2}\right)-\phi_{2}(x)}{2\left(x_{3}-x_{0}\right)\left(x_{2}-x_{0}\right)\left(x_{1}-x_{0}\right)},
\end{aligned}
$$




$$
\begin{aligned}
& \alpha_{1}(x)=\frac{x-x_{0}}{2\left(x_{1}-x_{0}\right)}-\frac{\left(x-x_{0}\right)\left(x-x_{1}\right)}{2\left(x_{2}-x_{0}\right)\left(x_{1}-x_{0}\right)}-\frac{\left(x-x_{0}\right)\left(x-x_{1}\right)}{2\left(x_{2}-x_{0}\right)\left(x_{2}-x_{1}\right)} \\
& -\frac{\omega_{2}(x)}{2\left(x_{3}-x_{1}\right)\left(x_{2}-x_{1}\right)}+\frac{\left(x-x_{0}\right)\left(x-x_{1}\right)\left(x-x_{2}\right)-\phi_{2}(x)}{2\left(x_{3}-x_{0}\right)\left(x_{3}-x_{1}\right)\left(x_{2}-x_{1}\right)} \\
& +\frac{\left(x-x_{0}\right)\left(x-x_{1}\right)\left(x-x_{2}\right)-\phi_{2}(x)}{2\left(x_{3}-x_{0}\right)\left(x_{2}-x_{0}\right)\left(x_{2}-x_{1}\right)} \\
& +\frac{\left(x-x_{0}\right)\left(x-x_{1}\right)\left(x-x_{2}\right)-\phi_{2}(x)}{2\left(x_{3}-x_{0}\right)\left(x_{2}-x_{0}\right)\left(x_{1}-x_{0}\right)}, \\
& \alpha_{2}(x)=\frac{\omega_{2}(x)-\omega_{3}(x)}{2\left(x_{4}-x_{2}\right)\left(x_{3}-x_{2}\right)}+\frac{\omega_{2}(x)}{2\left(x_{3}-x_{1}\right)\left(x_{2}-x_{1}\right)} \\
& +\frac{\omega_{2}(x)}{2\left(x_{3}-x_{1}\right)\left(x_{3}-x_{2}\right)}+\frac{\left(x-x_{0}\right)\left(x-x_{1}\right)}{2\left(x_{2}-x_{0}\right)\left(x_{2}-x_{1}\right)} \\
& -\frac{\left(x-x_{0}\right)\left(x-x_{1}\right)\left(x-x_{2}\right)-\phi_{2}(x)}{2\left(x_{3}-x_{0}\right)\left(x_{3}-x_{1}\right)\left(x_{3}-x_{2}\right)} \\
& -\frac{\left(x-x_{0}\right)\left(x-x_{1}\right)\left(x-x_{2}\right)-\phi_{2}(x)}{2\left(x_{3}-x_{0}\right)\left(x_{3}-x_{1}\right)\left(x_{2}-x_{1}\right)} \\
& -\frac{\left(x-x_{0}\right)\left(x-x_{1}\right)\left(x-x_{2}\right)-\phi_{2}(x)}{2\left(x_{3}-x_{0}\right)\left(x_{2}-x_{0}\right)\left(x_{2}-x_{1}\right)}, \\
& \alpha_{3}(x)=\frac{\omega_{3}(x)-\omega_{4}(x)}{2\left(x_{5}-x_{3}\right)\left(x_{4}-x_{3}\right)}-\frac{\omega_{2}(x)-\omega_{3}(x)}{2\left(x_{4}-x_{2}\right)\left(x_{4}-x_{3}\right)} \\
& -\frac{\omega_{2}(x)-\omega_{3}(x)}{2\left(x_{4}-x_{2}\right)\left(x_{3}-x_{2}\right)}-\frac{\omega_{2}(x)\left(x_{3}-x_{0}\right)+\phi_{2}(x)}{2\left(x_{3}-x_{1}\right)\left(x_{3}-x_{2}\right)\left(x_{3}-x_{0}\right)} \\
& +\frac{\left(x-x_{0}\right)\left(x-x_{1}\right)\left(x-x_{2}\right)}{2\left(x_{3}-x_{0}\right)\left(x_{3}-x_{1}\right)\left(x_{3}-x_{2}\right)} \\
& \alpha_{n-3}(x)=\frac{\omega_{n-5}(x)-\omega_{n-4}(x)}{2\left(x_{n-3}-x_{n-5}\right)\left(x_{n-3}-x_{n-4}\right)}-\frac{\omega_{n-4}(x)-\omega_{n-3}(x)}{2\left(x_{n-2}-x_{n-4}\right)\left(x_{n-3}-x_{n-4}\right)} \\
& -\frac{\omega_{n-4}(x)-\omega_{n-3}(x)}{2\left(x_{n-2}-x_{n-4}\right)\left(x_{n-2}-x_{n-3}\right)}+\frac{\omega_{n-3}(x)}{2\left(x_{n-1}-x_{n-3}\right)\left(x_{n-2}-x_{n-3}\right)} \\
& -\frac{\left(x-x_{n}\right)\left(x-x_{n-1}\right)\left(x-x_{n-2}\right)+\phi_{n-2}(x)}{2\left(x_{n}-x_{n-3}\right)\left(x_{n-1}-x_{n-3}\right)\left(x_{n-2}-x_{n-3}\right)}, \\
& \alpha_{n-2}(x)=\frac{\left(x-x_{n}\right)\left(x-x_{n-1}\right)}{2\left(x_{n}-x_{n-2}\right)\left(x_{n-1}-x_{n-2}\right)}-\frac{\omega_{n-3}(x)}{2\left(x_{n-1}-x_{n-3}\right)} \\
& +\frac{\left(x-x_{n}\right)\left(x-x_{n-1}\right)\left(x-x_{n-2}\right)+\phi_{n-2}(x)}{2\left(x_{n}-x_{n-3}\right)\left(x_{n}-x_{n-2}\right)\left(x_{n-1}-x_{n-2}\right)} \\
& +\frac{\left(x-x_{n}\right)\left(x-x_{n-1}\right)\left(x-x_{n-2}\right)+\phi_{n-2}(x)}{2\left(x_{n}-x_{n-3}\right)\left(x_{n-1}-x_{n-3}\right)\left(x_{n-1}-x_{n-2}\right)} \\
& +\frac{\omega_{n-4}(x)-\omega_{n-3}(x)}{2\left(x_{n-2}-x_{n-4}\right)\left(x_{n-2}-x_{n-3}\right)}-\frac{\omega_{n-3}(x)}{2\left(x_{n-1}-x_{n-3}\right)\left(x_{n-1}-x_{n-2}\right)} \\
& +\frac{\left(x-x_{n}\right)\left(x-x_{n-1}\right)\left(x-x_{n-2}\right)+\phi_{n-2}(x)}{2\left(x_{n}-x_{n-3}\right)\left(x_{n-1}-x_{n-3}\right)\left(x_{n-2}-x_{n-3}\right)}, \\
& \alpha_{n-1}(x)=-\frac{\left(x-x_{n}\right)}{2\left(x_{n}-x_{n-1}\right)}-\frac{\left(x-x_{n}\right)\left(x-x_{n-1}\right)}{2\left(x_{n}-x_{n-2}\right)\left(x_{n}-x_{n-1}\right)} \\
& -\frac{\left(x-x_{n}\right)\left(x-x_{n-1}\right)}{2\left(x_{n}-x_{n-2}\right)\left(x_{n-1}-x_{n-2}\right)}+\frac{\omega_{n-3}(x)}{2\left(x_{n-1}-x_{n-3}\right)\left(x_{n-1}-x_{n-2}\right)}
\end{aligned}
$$

$$
\begin{array}{r}
-\frac{\left(x-x_{n}\right)\left(x-x_{n-1}\right)\left(x-x_{n-2}\right)+\phi_{n-2}(x)}{2\left(x_{n}-x_{n-3}\right)\left(x_{n}-x_{n-2}\right)\left(x_{n}-x_{n-1}\right)} \\
-\frac{\left(x-x_{n}\right)\left(x-x_{n-1}\right)\left(x-x_{n-2}\right)+\phi_{n-2}(x)}{2\left(x_{n}-x_{n-3}\right)\left(x_{n}-x_{n-2}\right)\left(x_{n-1}-x_{n-2}\right)} \\
-\frac{\left(x-x_{n}\right)\left(x-x_{n-1}\right)\left(x-x_{n-2}\right)+\phi_{n-2}(x)}{2\left(x_{n}-x_{n-3}\right)\left(x_{n-1}-x_{n-3}\right)\left(x_{n-1}-x_{n-2}\right)}, \\
\alpha_{n}(x)=\frac{1}{2}+\frac{x-x_{n}}{2\left(x_{n}-x_{n-1}\right)}+\frac{\left(x-x_{n}\right)\left(x-x_{n-1}\right)}{2\left(x_{n}-x_{n-2}\right)\left(x_{n}-x_{n-1}\right)} \\
+\frac{\left(x-x_{n}\right)\left(x-x_{n-1}\right)\left(x-x_{n-2}\right)+\phi_{n-2}(x)}{2\left(x_{n}-x_{n-3}\right)\left(x_{n}-x_{n-2}\right)\left(x_{n}-x_{n-1}\right)},
\end{array}
$$

and

$$
\omega_{j}(x)=\frac{\phi_{j}(x)-\phi_{j+1}(x)}{x_{j+2}-x_{j-1}}, 1 \leq j \leq n-2 .
$$

By rewriting (3), we get another expression of $L_{R}(x)$ as follows

$$
\begin{aligned}
& L_{R}(x)=\frac{1}{2} \sum_{j=2}^{n-2} f\left[x_{j-2}, x_{j-1}, x_{j}, x_{j+1}, x_{j+2}\right]\left(x_{j+2}-x_{j-2}\right) \phi_{j}(x) \\
& +\frac{1}{2}\left\{f\left(x_{0}\right)+f\left[x_{0}, x_{1}\right]\left(x-x_{0}\right)+f\left[x_{0}, x_{1}, x_{2}\right]\left(x-x_{0}\right)\left(x-x_{1}\right)\right. \\
& \left.+f\left[x_{0}, x_{1}, x_{2}, x_{3}\right]\left(x-x_{0}\right)\left(x-x_{1}\right)\left(x-x_{2}\right)\right\} \\
& +\frac{1}{2}\left\{f\left(x_{n}\right)+f\left[x_{n}, x_{n-1}\right]\left(x-x_{n}\right)+f\left[x_{n}, x_{n-1}, x_{n-2}\right]\left(x-x_{n}\right)\left(x-x_{n-1}\right)\right. \\
& \left.+f\left[x_{n}, x_{n-1}, x_{n-2}, x_{n-3}\right]\left(x-x_{n}\right)\left(x-x_{n-1}\right)\left(x-x_{n-2}\right)\right\},
\end{aligned}
$$$$
\text { where } f\left[x_{j+1}, x_{j}, x_{j-1}\right] \text { denotes the divided difference of }
$$$$
\text { function } f(x) \text {. }
$$

\section{B. The properties of quasi-interpolation operator $L_{R}(x)$}

Similar to $[6,8]$, we can obtain the polynomial reproduction property and shape-preserving properties of the quasiinterpolation operator $L_{R}(x)$ in following results.

Theorem 1.1: Quasi-interpolation operator $L_{R}(x)$ satisfies the cubic polynomial reproduction property, i.e.,

$$
\begin{aligned}
& \sum_{j=0}^{n}\left(a_{0} x_{j}^{3}+a_{1} x_{j}^{2}+a_{2} x_{j}+a_{3}\right) \alpha_{j}(x) \\
& =a_{0} x^{3}+a_{1} x^{2}+a_{2} x+a_{3}, a_{i} \in R, i=0,1,2,3 .
\end{aligned}
$$

Theorem 1.2: If the points sequence (1) is uniformly distributed and the data sequence $\left\{f\left(x_{j}\right)\right\}_{j=0}^{n}$ stems from a convex function $f(x) \in C\left[x_{0}, x_{n}\right]$, then the quasi-interpolation operator $L_{R}(x)$ is also a quasi-convex function.

Theorem 1.3: The quasi-interpolation operator $L_{R}(x)$ is strict shape-preserving of order 3.

Theorem 1.4: The quasi-interpolation operator $L_{R}(x)$ is strict shape-preserving of order 4 . 


\section{The approximation order of the quasi-interpolation operator $L_{R}(x)$}

Similar to [8], we can obtain the convergence analysis to the approximation capacity of the quasi-interpolation operator $L_{R}(x)$.

Theorem 1.5: If the third order derivative of $f(x)$ is Lipschitz continuous, then the approximation capacity of $L_{R}(x)$ satisfies

$$
\left\|L_{R}(x)-f(x)\right\|_{\infty} \leq O\left(h^{4}\right),
$$

when $c=O\left(h^{2}\right)$.

\section{NUMERICAL SCHEME USING QUASI-INTERPOLATION}

In this section, we present the numerical scheme for solving diffusion equation by using the quasi-interpolation. First, we study the derivative of order $m=1,2,3$ of the approximation capacity of the quasi-interpolation operator $L_{R}(x)$.

Theorem 2.1: If the third order derivative of $f(x)$ is Lipschitz continuous, then the derivative of order $m=1,2,3$ of the approximation capacity of $L_{R}(x)$ satisfies $\left\|L_{R}^{(m)}(x)-f^{(m)}(x)\right\|_{\infty} \leq C_{0} h(h+c)^{3-m}+C_{1} c^{2}$.

Proof: For any fixed $x \in[A, B]$, let $p(y)$ be the local Taylor polynomial of $f(y)$ at the fixed point $x$, i.e. $p(y)=f(x)+f^{\prime}(x)(y-x)+\frac{1}{2} f^{\prime \prime}(x)(y-x)^{2}+\frac{1}{3 !} f^{\prime \prime \prime}(x)(y-x)^{3}$.

Based on Theorem 1.1, we have

$$
\begin{aligned}
& \left|L_{R}^{(m)}(x)-f^{(m)}(x)\right|=\left|\left[L_{R}(x)-f(x)\right]^{(m)}\right| \\
& =\left|\left[\sum_{j=0}^{n}\left[f\left(x_{j}\right)-p\left(x_{j}\right)\right] \alpha_{j}(x)\right]^{(m)}\right| \\
& \leq \frac{1}{2} \mid \sum_{j=2}^{n-2}\left\{f\left[x_{j-2}, x_{j-1}, x_{j}, x_{j+1}, x_{j+2}\right]\right. \\
& \left.-p\left[x_{j-2}, x_{j-1}, x_{j}, x_{j+1}, x_{j+2}\right]\right\}\left(x_{j+2}-x_{j-2}\right) \phi_{j}^{(m)}(x) \mid \\
& +C_{1}\left[\left(x-x_{0}\right)^{4}\right]^{(m)}+C_{2}\left[\left(x_{n}-x\right)^{4}\right]^{(m)} \\
& =\frac{1}{2} \mid \sum_{j=2}^{n-2}\left\{f\left[x_{j-1}, x_{j}, x_{j+1}, x_{j+2}\right]\right. \\
& \left.-f\left[x_{j-2}, x_{j-1}, x_{j}, x_{j+1}\right]\right\} \phi_{j}^{(m)}(x) \mid \\
& +C_{1}\left[\left(x-x_{0}\right)^{4}\right]^{(m)}+C_{2}\left[\left(x_{n}-x\right)^{4}\right]^{(m)} \\
& \leq \frac{1}{12} \sum_{j=2}^{n-2}\left|f^{(3)}\left(\xi_{j}\right)-f^{(3)}\left(\eta_{j}\right) \| \phi_{j}^{(m)}(x)\right| \\
& +C_{1}\left[\left(x-x_{0}\right)^{4}\right]^{(m)}+C_{2}\left[\left(x_{n}-x\right)^{4}\right]^{(m)} \\
& \left(\xi_{j} \in\left(x_{j-1}, x_{j+2}\right), \eta_{j} \in\left(x_{j-2}, x_{j+1}\right)\right)
\end{aligned}
$$

$\leq \frac{1}{3} C_{0} h \sum_{j=2}^{n-2}\left|\phi_{j}^{(m)}(x)\right|+C_{1}\left[\left(x-x_{0}\right)^{4}\right]^{(m)}+C_{2}\left[\left(x_{n}-x\right)^{4}\right]^{(m)}$

$\leq \frac{1}{3} C_{0} h \sum_{\left|x-x_{j}\right| \leq h}\left|\left[\phi_{j}(x)\right]^{(m)}\right|+\frac{1}{3} C_{0} h \sum_{\left|x-x_{j}\right|>h}\left|\left[\phi_{j}(x)\right]^{(m)}\right|$

$+C_{1}\left[\left(x-x_{0}\right)^{4}\right]^{(m)}+C_{2}\left[\left(x_{n}-x\right)^{4}\right]^{(m)}$

$\leq \frac{1}{3} C_{0} h \sum_{\left|x-x_{j}\right| \leq h}\left|\phi_{j}^{(m)}(x)\right|+\left[\frac{1}{3} C_{0} \int_{|x-t|>h} 2|x-t|\left[(x-t)^{2}+c^{2}\right] d t\right.$

$+C_{1}\left[\left(x-x_{0}\right)^{4}\right]^{(m)}+C_{2}\left[\left(x_{n}-x\right)^{4}\right]^{(m)}$

$\leq \frac{1}{3} C_{0} h(h+c)^{3-m}+C_{1}\left\{\left[\left(x-x_{0}\right)^{2}+c^{2}\right]^{2}-\left(x-x_{0}\right)^{4}\right\}^{(m)}$

$+C_{2}\left\{\left[\left(x_{n}-x\right)^{2}+c^{2}\right]^{2}-\left(x_{n}-x\right)^{4}\right\}^{(m)}$

$\leq C_{0} h(h+c)^{3-m}+C_{1} c^{2}$.

where $C_{i}, i=0,1$ are positive constants and independent of $x$ and $h$.

Let $\Omega=[A, B], I=[0, T]$, be space and time domain, respectively. We denote $\square_{T}:=\Omega \times I$. The one-dimensional diffusion equation we consider in this paper reads

$$
\frac{\partial u(x, t)}{\partial t}-\frac{\partial^{2} u(x, t)}{\partial x^{2}}=g(x, t), \forall(x, t) \in \square_{T},
$$

subject to the following initial and boundary conditions

$$
\begin{aligned}
& u(x, 0)=0, \forall x \in \Omega, \\
& u(A, t)=u(B, t)=0, \forall t \in I .
\end{aligned}
$$

Here $g(x, t)$ is a source term.

In space, we use the second derivative of the quasiinterpolation to approximate $\frac{\partial^{2} u\left(x_{j}, t_{k}\right)}{\partial x^{2}}$, thus

$$
\left[\frac{\partial^{2} u(x, t)}{\partial x^{2}}\right]_{j}^{k}=\frac{\partial^{2} u\left(x_{j}, t_{k}\right)}{\partial x^{2}}=\sum_{l=0}^{k} u_{l}^{k} \frac{\partial^{2} \alpha_{l}\left(x_{j}\right)}{\partial x^{2}} \square\left[L_{R}^{(2)}(x, t)\right]_{j}^{k},
$$

In time, we use Crank-Nicolson scheme and back Euler scheme, then we get

$$
\begin{aligned}
\frac{u_{j}^{k+1}-u_{j}^{k}}{\Delta t}= & \frac{1}{2}\left\{\left[L_{R}^{(2)}(x, t)\right]_{j}^{k+1}+\left[L_{R}^{(2)}(x, t)\right]_{j}^{k}\right\}+g_{j}^{k+\frac{1}{2}} . \\
& \frac{u_{j}^{k+1}-u_{j}^{k}}{\Delta t}=\left[L_{R}^{(2)}(x, t)\right]_{j}^{k+1}+g_{j}^{k+1} .
\end{aligned}
$$

where $u_{j}^{k}$ is the approximation of the value of $u\left(x_{j}, t_{k}\right)$ and $g_{j}^{k}=g\left(x_{j}, t_{k}\right), t_{k}=k \Delta t ; \Delta t$ is time step.

Based on truncation error of Crank-Nicolson scheme and back Euler scheme in time and the approximation capacity of the quasi-interpolation operator $L_{R}(x)$ (see Theorem 2.1) in space, we obtain following result for $c=O\left(h^{2}\right)$.

Theorem 2.2: (1) The truncation error of the scheme (10) is of order $O\left(\Delta t^{2}+h^{2}\right)$. (2) The truncation error of the scheme (11) is of order $O\left(\Delta t+h^{2}\right)$.

\section{NUMERICAL EXAMPLES}

We test the algorithm by two parts. The first part, we test the quasi-interpolation. Suppose $f(x)=x^{4}$ is an approximated function, then we choose shape parameter $c=h^{2}$ to obtain 
the approximation capacity of our quasi-interpolation operator $L_{R}(x)$. The results are shown in Figure 1.

The second part, we test the diffusion equation. We consider the problem (7)-(9) with an exact analytical solution

$$
u(x, t)=t^{2} \sin (2 \pi x) \text {. }
$$

It can be checked that the corresponding forcing term

$$
g(x, t)=2 t\left(1+2 \pi^{2} t\right) \sin (2 \pi x) .
$$

The numerical results reported in the Tables below have been evaluated at $\Omega=[0,1]$ and $T=1$. Figures 2 and Figures 3 are show the error of the scheme (10) and scheme (11), respectively. From Figures 2-3, we find the numerical solutions are acceptable. Furthermore, we find the error become small as the parameter $c$ becoming small.

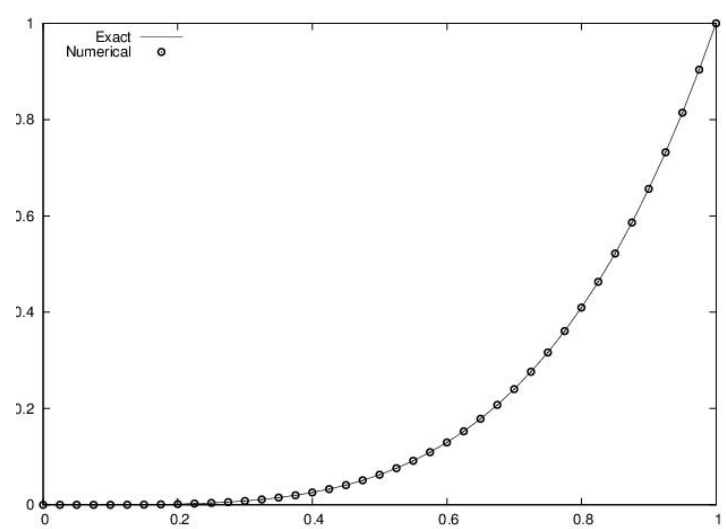

Fig. 1. The function $f(x)=x^{4}$ and its quasi-interpolation $L_{R}(x)$.

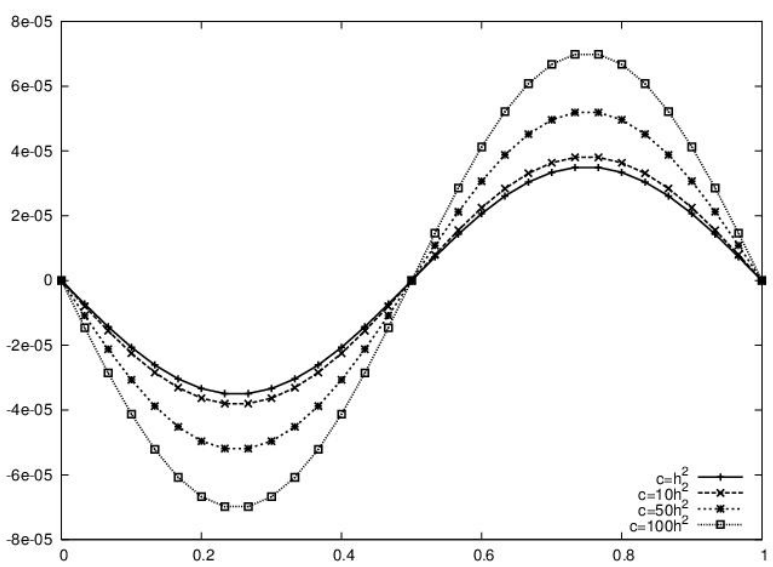

Fig. 2. The error function with $h=\frac{1}{300}, \Delta t=\frac{1}{10000}$ for scheme (10).

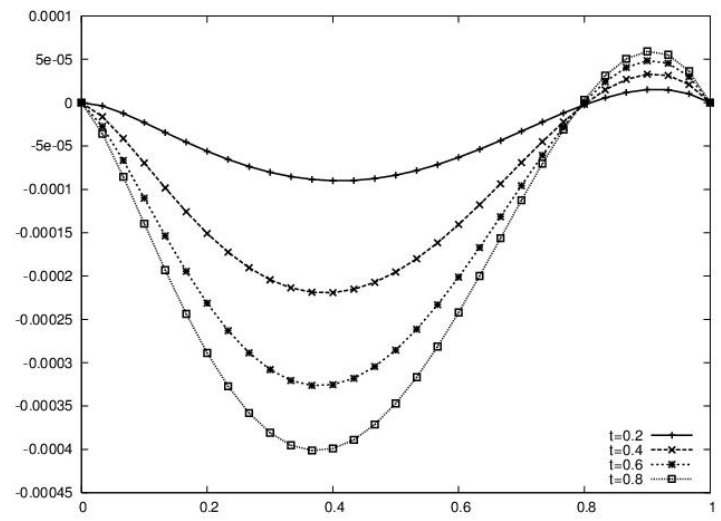

Fig. 3.The error function with $h=\frac{1}{300}, \Delta t=\frac{1}{10000}$ for scheme (11).

\section{ACKNOWLEDGMENT}

The authors would like to thank the National Natural Science Foundation of China (Grant No.90916027), Foundation of Guizhou Science and Technology Department (Grant No.[2013]2144) and also supported by 2013 Key project of scientific research project of Guizhou Minzu University.

\section{REFERENCES}

[1] R. Schaback and Z.Wu, "Operators on radial functions," J. Comput. Appl. Math., vol.73,no.1,pp.257-270, 1996.

[2] WA Light, "Some aspects of radial basis function approximation," Approx. Theory, Spline Functions and Applications, vol. 356,pp.163$190,1992$.

[3] R.L. Hardy, "Multiquadric equations of topography and other irregular surfaces," J. Geophys. Res., vol.76,no.8,pp.1905-1915,1971.

[4] R. Franke, "Scattered Data Interpolation: Tests of Some Methods," Math. Comput., vol.38,no.157,pp.181-200,1982.

[5] L. Ling, "A univariate quasi-multiquadric interpolationwith better smoothness," Comput. Math. Appl., vol.48,no.5-6,pp.897-912,2004.

[6] R.K Beatson and M.J.D. Powell, "Univariate multiquadric approximation: quasi-interpolation to scattered data," Constr. Approx., vol.8,no.3,pp.275-288,1992.

[7] W. Zhang and Z. Wu, "Some shape-preserving quasi-interpolants to nonuniformly distributed data by MQ-B-splines," Appl. Math. J. Chinese Univ. Ser. A, vol.19,no.2,pp.191-202,2004.

[8] R. Chen and Z. Wu, "Solving hyperbolic conservation laws using multiquadric quasi-interpolation," Numer. Methods Partial Differential Equations, vol.22,no.4,pp.776-796, 2006.

[9] M. Tatari and M. Dehghan, "A method for solving partial differential equations via radial basis functions: Application to the heat equation," Eng. Anal. Bound. Elem., vol.34,no.3,pp.206-212,2010.

[10] C.G. Zhu and R.H. Wang, "Numerical solution of Burgers' equation by cubic B-spline quasi-interpolation," Appl. Math. Comput., vol.208,no.1,pp.260-272,200

[11] Y. Duan and F. Rong, A numerical scheme for nonlinear Schrǒdinger equation by MQ quasi-interpolation, Engineering Analysis with Boundary Elements, vol37,no 1,pp.:89-94,2013 